\title{
Comparing Voice Self-Assessment with Auditory Perceptual Analysis in Patients with Multiple Sclerosis
}

\author{
Vladimir Bauer $^{1}$ Zorica Aleric ${ }^{1}$ Ervin Jancic ${ }^{2}$ \\ ${ }^{1}$ Department of Otorhinolaryngology, General Hospital Karlovac, \\ Karlovac, Croatia \\ 2 Department of Neurology, General Hospital Karlovac, \\ Karlovac, Croatia
}

Address for correspondence Vladimir Bauer, MD, Department of Otorhinolaryngology, General Hospital Karlovac, A Stampara 3, Karlovac, Karlovac 47000, Croatia (e-mail: vbauermail@yahoo.com).

Int Arch Otorhinolaryngol 2015;19:100-105.

\begin{abstract}
Introduction Disordered voice quality could be a symptom of multiple sclerosis (MS). The impact of MS on voice-related quality of life is still controversial.

Objectives The aim of this study was to compare the results of voice self-assessment with the results of expert perceptual assessment in patients with MS.

Methods The research included 38 patients with relapse-remitting MS ( 23 women and 15 men; ages 21 to 83, mean $=44$ ). All participants filled out a Voice Handicap Index (VHI), and their voice sample was analyzed by speech and language professionals using the Grade Roughness Breathiness Asthenia Strain scale (GRBAS).

Results The patients with MS had significantly higher VHI than control group participants (mean value $16.68 \pm 16.2$ compared with $5.29 \pm 5.5, p=0.0001$ ). The study established a notable level of dysphonia in $55 \%$, roughness and breathiness in $66 \%$, asthenia in $34 \%$, and strain in $55 \%$ of the vocal samples. A significant correlation was established between VHI and GRBAS scores $(r=0.3693, p=0.0225)$, and $\mathrm{VHI}$ and asthenia and strain components $(r=0.4037$ and $0.3775, p=0.012$ and 0.0195 , respectively). The female group showed positive and significant correlation between claims for self-assessing one's voice (pVHI) and overall GRBAS scores, and between pVHI

\section{Keywords}

- multiple sclerosis

- quality of life

- voice quality

- voice disorders and grade, roughness, asthenia, and strain components. No significant correlation was found for male patients $(p>0.05)$.

Conclusion A significant number of patients with MS experienced voice problems. The $\mathrm{VHI}$ is a good and effective tool to assess patient self-perception of voice quality, but it may not reflect the severity of dysphonia as perceived by voice and speech professionals.
\end{abstract}

\section{Introduction}

Multiple sclerosis (MS) is a neurologic disease with symptoms that affect various organ systems. Voice and speech are affected in $44 \%$ of patients with MS. ${ }^{1}$ Speech disorders are much more researched than voice disorders. Voice disorders are diagnosed using objective diagnostic methods. Phonatory instability was proved through acoustic voice analysis ${ }^{2}$ and through electroglottography. ${ }^{3}$ Spectral voice analysis showed that $70 \%$ of patients had symptoms of dysphonia compared with $33 \%$ of patients in the control group. The changes were more notable among men than women. The basic frequency, basic frequency deviation, and jitter level are all higher. ${ }^{4}$ Even more importantly, objective voice changes were described by received

July 13,2014

accepted

November 14, 2014

published online

December 30, 2014
DOI http://dx.doi.org/

$10.1055 / \mathrm{s}-0034-1397332$. ISSN $1809-9777$.
Copyright $\odot 2015$ by Thieme Publicações License terms

Ltda, Rio de Janeiro, Brazil
(®) $\Theta \circledast$ 
Dogan et al. $^{5}$ In their sample, more significant differences between the MS group and a control group were observed for jitter, shimmer, soft phonation index, and maximal phonation time. Using videostroboscopy, 16 of 27 patients showed incomplete glottal closure as an objective sign of vocal cord dysfunction in phonation. ${ }^{5}$ All these and other studies claim that many patients with MS suffer from vocal changes that can be detected and measured through diagnostic methods. $^{6-8}$

Some research has dealt with the subjective experience of vocal changes and the impact of these changes on the quality of life, but the results are conflicting. Dogan et al used the Voice Handicap Index (VHI) as a method for self-assessing vocal problems, ${ }^{5}$ but the authors did not describe the results in detail. Instead, they only concluded that the results were not significantly different in patients with MS compared with the control group. The same conclusion was given in the study where only $12 \%$ of patients with MS described the difficulties of dysphonia. ${ }^{9}$ On the other hand, the study of patients with MS performed by Natour showed significant differences in the self-assessment of vocal difficulties. ${ }^{10}$ VHI was used, and a significant difference was observed between the total score and all subscores that were higher for patients with MS than the control group. The conclusion of this study is that MS can make communication more difficult and cause a high sense of vocal handicap. In our pervious study, ${ }^{11}$ the VHI score was significantly higher in the MS group. According to the study, ${ }^{12}$ the level of 12 points in the VHI test should be considered as threshold for rating the biopsychosocial impact of dysphonia. Compared with previously mentioned results, $44 \%$ of patients with MS had a total VHI score higher than 12 (38\% of male patients and $48 \%$ of female patients). It is comparable with other previously mentioned results. ${ }^{2,3}$ Our present study is based on the discrepancies regarding prevalence and impact of the voice changes in MS. We completed and equalized our patient sample, and we included only patients in relapseremitted form of MS. The aim of this study is to compare the results of voice self-assessment with the results of expert perceptual assessment. It is our assumption that vocal difficulties reported by patients with MS will be confirmed and rated by a listener, a speech and vocal therapist.

\section{Methods}

This is a cross-sectional case-control study, in which voices of participants with and without MS were evaluated by selfperception questionnaire and hearing perceptual evaluation. Patients with MS were selected randomly from a list of patients attending Neurology Polyclinic for their routine follow-up visits, using the Hospital Information System database. The control group was composed of individuals without history of neurologic disease and disorder of communication recruited from hospital staff and patients' companions. Exclusion criteria were endotracheal intubation within 3 months before entry, history of laryngeal malignancy, an operation performed on the larynx and vocal chords, or an acute upper airway respiratory infection. The institutional Medical Ethical Committee approved the research.
All participants were administered the VHI, a standardized 30 -point questionnaire suggested by Jacobson et al in 1997, ${ }^{13}$ to determine the effect of vocal problems on the quality of life. This is a commonly used method of biopsychosocial selfassessment of vocal difficulties. Each answer is graded 0 to 4 depending on the frequency of the difficulty: $0=$ no difficulties, 1 = almost never, 2 = sometimes, 3 = almost always, 4 = always. The questionnaire is divided into three subscales; the first refers to claims for self-assessing one's own voice (pVHI), the second refers to statements regarding the effect of voice in daily functions (fVHI), and the third refers to the effect of vocal difficulties on the participants' emotional status $(\mathrm{eVHI})$. We used the Croatian version of the VHI. ${ }^{14}$

Hearing perceptual voice evaluation was performed using GRBAS scale according to the Japanese Society of Logopedics and Phoniatrics. ${ }^{15}$ The voice of each participant reading a standard text for 2 minutes was recorded on tape. The voice sample was collected in a quiet room with the microphone positioned $5 \mathrm{~cm}$ away from the lips. Voice recordings were assessed by three experienced speech therapists in a doubleblind, randomized fashion. The examiners assess the grades of dysphonia $(G)$, roughness $(R)$, breathiness (B), asthenia $(A)$, and strain $(S)$ by listening to patient's voice, using a scale from 0 to 3 where $0=$ regular, $1=$ mildly pronounced, $2=$ moderate, 3 = very pronounced.

The Expanded Disability Status Score (EDSS), a standardized system for assessing neurologic deficit based on the grade of nine functional systems in which a score of 0 is a state with no neurologic manifestations and 10 is death by disease, ${ }^{16}$ was determined for all patients.

Statistical analysis was performed using free statistical software. ${ }^{20}$ The regularity of data distribution was tested using the Kolmogorov-Smirnov test. The VHI scores were not normally distributed. We used descriptive statistical methods to show features of groups and subgroups-mean values and standard deviation and median and interquartile range for data with irregular distribution. We used nonparametric tests to measure differences in significance between the groupschi-square for category variables and the Mann-Whitney test for the numerical data. The correlation between groups of participants was tested by the Spearman rank test. The level of significance was set to $p<0.05$.

\section{Results}

The research included 38 patients with MS, 23 women and 15 men, ages 21 to 83 (mean $=44$ ). The mean length of disease was 12.8 years (range 1 to 48 ). All participants had relapsing-remitting type of disease. We compared them with participants from a control group ( $n=38,21$ women, 17 men) of corresponding age and without signs of MS and voice disorders. A detailed overview of participant features is shown in - Table 1. The patients with MS had significantly higher VHI than control group participants (mean value $16.68 \pm 16.2$ compared with $5.29 \pm 5.5, p=0.0001$ ). The pVHI, fVHI, and eVHI values were significantly higher for patients with MS. When observed separately, women with MS had higher VHI values than men (18.39 compared with 
Table 1 Participant characteristics

\begin{tabular}{|l|l|l|}
\hline & Patients & Control group \\
\hline Total $n$ & 38 & 38 \\
\hline Male/female & $15 / 23$ & $17 / 21$ \\
\hline Age (mean/range) & $44 / 21-83$ & $43 / 18-72$ \\
\hline $\begin{array}{l}\text { Duration of disease, y } \\
\text { (mean/range) }\end{array}$ & & \\
\hline All patients & $12.7 / 1-48$ & \\
\hline Male & $12.9 / 1-31$ & \\
\hline Female & $12.6 / 2-48$ & \\
\hline EDSS (mean \pm SD) & & \\
\hline All patients & $2.7 \pm 1.06$ & \\
\hline Male & $2.54 \pm 0.96$ & \\
\hline Female & $2.78 \pm 1.1$ & \\
\hline
\end{tabular}

Abbreviations: EDSS, Expanded Disability Status Score; SD, standard deviation.

13.84). If we take 12 points as a cutoff point in the VHI test, $47 \%$ of all patients ( $46 \%$ of men and $49 \%$ of women) reported noticeable voice difficulties. Detailed results of the pVHI are described in - Table 2.

All components of the GRBAS scale were significantly expressed in the patient group ( - Table 3 ). The researchers established a significant level of dysphonia $(G=1,2$, or 3 ) in $55 \%$, roughness and breathiness in $66 \%$, asthenia in $34 \%$, and strain in $55 \%$ of vocal samples, and $34 \%$ of participants had one component of the GRBAS scale graded as severe.

- Table 4 shows correlation values for VHI self-assessment and GRBAS scale auditory perceptual assessment. A significant positive correlation was established for all participants between VHI and GRBAS scores $(r=0.3693, p=0.0225)$, and VHI and A and S components ( $p=0.012$ and 0.0195 , respectively). If we observe only $\mathrm{pVHI}$, the part that refers to sense of vocal difficulties, the correlations are even more significant $(r=0.42$ to 0.53 ; - Table 4). This was observed both for male and female patients. No significant correlation was found for male patients $(p>0.05)$. The female group showed positive and significant correlation between pVHI and overall GRBAS scores, and between pVHI and G, R, A, and S components (-Table 4).
All our participants had relatively mild functional difficulties (EDSS $=2.7 \pm 1.06$ ). The results of self-assessment and perceptive assessment could not be related with the extent of neurologic deficit (expressed with EDSS), nor with the duration of the disease. Among all the observed parameters, a significant correlation was observed only between EDSS and A component of the GRBAS scale $(r=0.6, p=0.0025)$ and between the duration of the disease and the A component of the GRBAS scale $(r=0.53, p=0.0007)$, only for female patients.

\section{Discussion}

Phonation is not just an expiration that produces vibrations but a complex physiologic process that includes a coordinated cooperation of multiple organ systems. The nervous system is especially involved. The laryngeal part of the phonatory function can be disrupted by the damages on several levels of the nervous system. The cranial nerve $X$ (i.e., the vagus nerve) is responsible for direct motor innervation of the intrinsic laryngeal muscles. Its fibers stem from the bodies of cells located in the medullary reticular formation in the nucleus ambiguus. The neuropathology that may affect the

Table 2 Results obtained using VHI in patients with multiple sclerosis and control group

\begin{tabular}{|l|l|l|l|}
\hline & Patients & Control group & $p$ value \\
\hline Total VHI, mean (range) & $16.68(0-85)$ & $5.29(0-20)$ & 0.0001 \\
\hline fVHI, mean (range) & $4.58(0-25)$ & $1.34(0-10)$ & 0.001 \\
\hline pVHI, mean (range) & $9.22(0-36)$ & $3.55(0-6)$ & 0.001 \\
\hline eVHI, mean (range) & $2.88(0-24)$ & $0.4(0-2)$ & 0.004 \\
\hline Total VHI male, mean (range) & $13.85(0-48)$ & $4.61(0-16)$ & 0.055 \\
\hline Total VHI female, mean (range) & $18.39(0-85)$ & $5.42(0-20)$ & 0.001 \\
\hline
\end{tabular}

Abbreviations: eVHI, emotional subscale; fVHI, functional subscale; pVHI, physical subscale; VHI, Voice Handicap Index. 
Table 3 Results using GRBAS scale in patients with multiple sclerosis patients and control group

\begin{tabular}{|c|c|c|c|c|c|c|c|c|c|c|c|}
\hline & \multicolumn{5}{|c|}{ Patients $(n=38)$} & \multicolumn{5}{|c|}{ Control group $(n=38)$} & \multirow[b]{2}{*}{$p$ value } \\
\hline & 0 & 1 & 2 & 3 & All & 0 & 1 & 2 & 3 & All & \\
\hline G & 17 & 2 & 17 & 2 & 38 & 28 & 7 & 2 & 1 & 38 & 0.0025 \\
\hline$R$ & 13 & 1 & 21 & 3 & 38 & 29 & 4 & 4 & 1 & 38 & 0.0008 \\
\hline$B$ & 13 & 2 & 23 & 0 & 38 & 32 & 1 & 4 & 1 & 38 & 0.0002 \\
\hline A & 25 & 1 & 7 & 5 & 38 & 33 & 3 & 2 & 0 & 38 & 0.0196 \\
\hline$S$ & 17 & 2 & 16 & 3 & 38 & 32 & 2 & 3 & 1 & 38 & 0.0071 \\
\hline
\end{tabular}

Abbreviations: A, asthenia; B, breathiness; $G$, grade; R, roughness; S, strain.

voice can be located even more centrally. The vagus nerve nuclei are affected by the pyramidal and extrapyramidal system. The pyramidal system establishes its influence through the corticobulbar tract and the extrapyramidal system through the basal ganglia. As a demyelinating process, MS can cause lesions at any height of the nervous system, subcortically in the white matter; in the cores of the brainstem; in the medulla oblongata, cerebellum, peripheral nerves; in the already mentioned vagus nerve; and in nerves that participate in respiration. ${ }^{17}$ With such complex

Table 4 Correlation of auditory perception with self-assessment and duration of the disease

\begin{tabular}{|c|c|c|c|c|c|c|}
\hline & GRBAS & G & $\mathbf{R}$ & B & A & $S$ \\
\hline \multicolumn{7}{|l|}{ All } \\
\hline \multicolumn{7}{|l|}{$\mathrm{VHI}$} \\
\hline$r$ & $0.3693^{a}$ & 0.2379 & 0.1975 & 0.1058 & $0.4037^{\mathrm{a}}$ & $0.3775^{a}$ \\
\hline$p$ value & $0.0225^{a}$ & 0.1503 & 0.2346 & 0.5272 & $0.012^{\mathrm{a}}$ & $0.0195^{\mathrm{a}}$ \\
\hline \multicolumn{7}{|l|}{$\mathrm{pVHI}$} \\
\hline$r$ & $0.5289^{a}$ & $0.3408^{a}$ & 0.2503 & 0.0601 & $0.4433^{a}$ & $0.4209^{a}$ \\
\hline$p$ value & $0.0006^{a}$ & $0.0363^{a}$ & 0.1295 & 0.72 & $0.0053^{a}$ & $0.0085^{a}$ \\
\hline \multicolumn{7}{|c|}{ Duration } \\
\hline$r$ & 0.0098 & 0.2265 & 0.1008 & 0.0497 & $0.5279^{a}$ & 0.1589 \\
\hline$p$ value & 0.9532 & 0.1715 & 0.547 & 0.7669 & $0.0007^{a}$ & 0.3407 \\
\hline \multicolumn{7}{|l|}{ Male } \\
\hline \multicolumn{7}{|l|}{$\mathrm{pVHI}$} \\
\hline$r$ & 0.1822 & 0.2038 & 0.0284 & 0.0462 & 0.3368 & 0.2993 \\
\hline$p$ value & 0.5157 & 0.4663 & 0.92 & 0.8701 & 0.2196 & 0.2785 \\
\hline \multicolumn{7}{|c|}{ Duration } \\
\hline$r$ & 0.0359 & 0.1242 & 0.0021 & 0.2713 & 0.4442 & 0.0062 \\
\hline$p$ value & 0.8989 & 0.6593 & 0.9939 & 0.3281 & 0.0972 & 0.9825 \\
\hline \multicolumn{7}{|l|}{ Female } \\
\hline \multicolumn{7}{|l|}{$\mathrm{pVHI}$} \\
\hline$r$ & 0.7056 & $0.633^{a}$ & $0.4597^{a}$ & 0.182 & $0.4888^{a}$ & $0.3994^{a}$ \\
\hline$p$ value & $0.0002^{\mathrm{a}}$ & $0.0012^{\mathrm{a}}$ & $0.0273^{a}$ & 0.4059 & $0.0179^{a}$ & $0.059^{a}$ \\
\hline \multicolumn{7}{|c|}{ Duration } \\
\hline$r$ & 0.0301 & 0.2543 & 0.1366 & 0.1118 & $0.5777^{a}$ & 0.2383 \\
\hline$p$ value & 0.8916 & 0.2416 & 0.5341 & 0.6117 & $0.0039^{a}$ & 0.2736 \\
\hline
\end{tabular}

Abbreviations: A, asthenia; B, breathiness; G, grade; GRBAS, Grade Roughness Breathiness Asthenia Strain scale total score; pVHI, physical subscale of Voice Handicap Index; R, roughness; S, strain; VHI, Voice Handicap Index.

${ }^{a}$ Marked values correlated significantly. 
possibilities of emergence of dysphonia in MS, the psychological, social, and emotional importance of voice contributes to the importance and the complexity of its effect on the quality of life. Our research shows that patients with MS display more intense vocal difficulties than people without MS. The VHI score was above 12 for $47 \%$ of the participants.

The quality of life for patients with MS is a common research subject. Although objective estimates of vocal changes are well researched and objectively confirm symptoms of dysphonia in 30 to $70 \%$ of participants, ${ }^{2-8}$ little is known about the effect of dysphonia on the quality of life in MS. The research done on 27 female patients with MS used VHI and GRBAS. ${ }^{5}$ The pVHI showed no significant difference between the patients and the control group. The results of the GRBAS scale showed markedly higher A and S components in the MS group ( $p<0.0001$ for $A$ and $p=0.04$ for $S$ ). Although expressed somewhat more often with patients, the $G, R$, and $B$ component were not significantly different. A study of 59 participants with MS found no significant differences in the overall VHI score and subscores compared with the scores of healthy participants $(\mathrm{VHI}=5.9, \mathrm{pVHI}=2.4, \mathrm{fVHI}=2.2$, and $\mathrm{eVHI}=1.4) .{ }^{9}$ Only $8.7 \%$ of participants had serious vocal difficulties (VHI > 15). Contrary to that, the work by Natour et al analyzed VHI with 39 patients with MS, and a significant difference between patients with MS and healthy participants was noticed for both male and female participants (male VHI $=27.7$ compared with $\mathrm{VHI}=10.1$ in the control group and female $\mathrm{VHI}=19$ compared with $\mathrm{VHI}=11.4$ in the control group). ${ }^{10}$ The difference was significant for total score and all three subscores. These scores, along with those from our research, can be compared according to their similarities and differences. All four studies used the VHI scale, and two used the GRBAS scale. The results of self-assessed VHI differ greatly. Some of that difference can be explained by the various degrees of total neurologic damage. Studies describing insignificant differences have participants with a relatively low EDSS score: 1.94 (8) and 1.22 (5), although mean EDSS score for our study was 2.7. A score of 1.5 to 2 is interpreted as a state without neurologic incidents, with only minimal signs of damage to the functional systems. A score of 2.5 to 3 implies mild incidents in the two functional systems. Not only does higher EDSS imply more total damage, the incidents, no matter how gentle, additionally sensitize the participants to other difficulties that the EDSS does not measure. Although the duration of the disease was correlated with either VHI or GRBAS scale in our study, it should be noted that there is a difference in disease duration between our sample and patients from the previously mentioned studies (12 years compared with 6 and 7 years). Linguistic and cultural differences among the participants, such as being speakers of various languages, could affect the perception of vocal difficulties and were not the subject of our research.

To make our self-assessment results more valuable, they were complemented with the results of perceptual voice assessment. Perceptual evaluation is fundamental in assessing voice quality, the relevance of defects, and the defects' impact on the subject's ability to communicate. We tried to find the differences in the vocal perceptions between the participants expressed by VHI and by the listeners expressed by the GRBAS scale. Because the VHI is divided into three subscales, we expected the biggest congruence between the physical aspect (pVHI), which describes the perception of vocal difficulties, and the GRBAS scale. Smaller or no congruence was expected for the fVHI and eVHI. We found no significant correlation between overall VHI or its subscales and any of the GRBAS scale components for male participants. We found significant positive correlation between $\mathrm{VHI}$ and pVHI with the GRBAS overall score and the components $G, B$, $A$, and $S$ among female participants. We found very few studies that compared self-assessment with the perceptual assessment for patients with MS. The results were somewhat comparable with an already mentioned study, ${ }^{5,11}$ in which $\mathrm{A}$ and $\mathrm{S}$ components of the GRBAS scale were graded significantly different for female patients with MS. In this case, a lower EDSS score could explain the differences between the expression of $\mathrm{G}$ and $\mathrm{B}$ components of the GRBAS scale (1.2 compared with 2.7). We cannot explain why the VHI result for male participants is not in correlation with the perceptual analysis. The VHI is a good and effective tool to assess a patient's self-perception of the voice quality, but it may not reflect the severity of dysphonia as perceived by voice and speech professionals. It seems that components other than those directly related to voice quality may contribute to responses on voice evaluation completed by patients. Gender is one of these. Certainly the gender of patients is a factor, but we assumed that the gender of listeners also could be a factor. All of our three voice professionals were female, possibly more sensitized on voices of their own gender. In any case, the research should be repeated on a large number of male participants using several questionnaires regarding the quality of voice and assessors of both genders. The same degree of dysphonia can impact different patients in very different ways. Factors such as duration of the disorder and age and social setting of the patient, along with the patient's occupation status, profession, general health, or other factors, may act to blunt or accentuate the effect of dysphonia upon a particular patient's voice related quality of life. However, important clinical information may be missed if only the patient's perspective is considered. A perceptual assessment tool such as the GRBAS scale gives valuable information about the extent of altered vocal physiology.

One parameter in significant correlation with total neurologic deficit and the disease length is the asthenia component of the GRBAS scale. Respiratory function is often affected by MS. Weakness of respiratory muscles is characterized by lowered maximal expiratory pressure ${ }^{7,10}$; weakness has a direct effect on the EDSS value and worsens with time. A maintained expiratory pressure enables adequate subglottic pressure and thus the movement of vocal cords and high quality of produced voice. Otherwise, the voice is weak and asthenic. Incomplete glottal closure, described as common in MS by Dogan et al, ${ }^{5}$ as well as posterior glottal chink, definitely contributes to vocal asthenia. The proportion of our participants with expressed subjective vocal difficulties ( VHI $=47 \%$, GRBAS $=55 \%$ ) is comparable to the ratio of dysphonic voices in the studies that used objective vocal analyses (30 to $70 \%$ ). ${ }^{3-5}$ 
Therefore, it is justifiable to think that a significant number of patients with MS have and feel vocal difficulties. It is obvious that the effect of vocal difficulties on the quality of life is usually not strong. VHI is markedly different than that of control group participants, but in most cases it implies mild difficulties. Only three participants from our research rated their VHI as moderately altered, and only one patient as severely altered $(\mathrm{VHI}=85)$. Unfortunately, MS is a disease with deficits that strongly affect mobility, independence, bladder control, sexual function, among others. When compared with the difficulties described previously, it is easy to see that vocal difficulties would get more attention if they were an isolated vocal disorder.

It is well known that MS can lead to hearing impairment. Moderate hearing loss occurs in $23 \%$ of patients, ${ }^{18}$ and difficulty swallowing is seen in $43 \%$ of patients with MS. ${ }^{19}$ Vocal disorders, speech, swallowing, and hearing difficulties can cause significant problems and disrupt the quality of life, especially when occurring simultaneously. Some of the previously mentioned symptoms could be the first signs of the disease and a reason to visit the otorhinolaryngologist. Although MS is not a primary otorhinolaryngologic disorder, it should be included in a differential diagnostic process.

A lack of objective measurements limits our study. However, we focused on the patients' experience of the quality of their voice compared with subjective analysis performed by voice professionals. We had a small number of patients $(n=38)$; we plan further research with a larger number of patients, more parameters, and comparisons with other nonmotor dysfunctions in patients with MS.

\section{Conclusion}

In our study, almost half of patients with MS feel and describe mild dysphonic difficulties that affect the quality of life. The perception of dysphonic difficulties is individual, not always in accordance with the hearing perceptual vocal analysis. Positive moderate correlation between self-assessment and perceptual vocal assessment was found for our female patients with MS.

\section{References}

1 Hartelius L, Svensson P. Speech and swallowing symptoms associated with Parkinson's disease and multiple sclerosis: a survey. Folia Phoniatr Logop 1994;46(1):9-17

2 Hartelius L, Buder EH, Strand EA. Long-term phonatory instability in individuals with multiple sclerosis. J Speech Lang Hear Res 1997;40(5):1056-1072
3 Konstantopoulos K, Vikelis M, Seikel JA, Mitsikostas DD. The existence of phonatory instability in multiple sclerosis: an acoustic and electroglottographic study. Neurol Sci 2010; 31(3):259-268

4 Feijó AV, Parente MA, Behlau M, Haussen S, de Veccino MC, Martignago BCDF. Acoustic analysis of voice in multiple sclerosis patients. J Voice 2004;18(3):341-347

5 Dogan M, Midi I, Yazici MA, Kocak I, Günal D, Sehitoglu MA. Objective and subjective evaluation of voice quality in multiple sclerosis. J Voice 2007;21(6):735-740

6 Duranovic M, Salihovic N, Ibrahimagic A. Characteristics of voice in individuals with multiple sclerosis. Mater Sociomed 2011;23(1): 23-27

7 Chiara T, Martin D, Sapienza C. Expiratory muscle strength training: speech production outcomes in patients with multiple sclerosis. Neurorehabil Neural Repair 2007;21(3):239-249

8 Yamout B, Fuleihan N, Hajj T, et al. Vocal symptoms and acoustic changes in relation to the expanded disability status scale, duration and stage of disease in patients with multiple sclerosis. Eur Arch Otorhinolaryngol 2009;266(11):1759-1765

9 Hamdan AL, Farhat S, Saadeh R, El-Dahouk I, Sibai A, Yamout B. Voice-related quality of life in patients with multiple sclerosis. Autoimmune Dis 2012;2012:143813. doi: 10.1155/2012/143813

10 Natour Y, Marie B, Aljunidy L. The respiratory muscle capabilities of Jordanian patients with multiple sclerosis. J Voice 2012;26(6): 811.e15-811.e18

11 Bauer V, Aleric Z, Jancic E, Knezevic B, Prpic D, Kacavenda A. Subjective and perceptual analysis of voice quality and relationship with neurological disfunction in multiple sclerosis patients. Clin Neurol Neurosurg 2013;115(Suppl 1):S17-S20

12 Niebudek-Bogusz E, Kuzańska A, Woznicka E, Sliwinska-Kowalska M. Assessment of the voice handicap index as a screening tool in dysphonic patients. Folia Phoniatr Logop 2011;63(5): 269-272

13 Jacobson BH, Johnson A, Grywalski C, et al. The Voice Handicap Index (VHI): development and validation. Am J Speech Lang Pathol 1996;6:66-70

14 Bonetti A, Bonetti L. Cross-cultural adaptation and validation of the Voice Handicap Index into Croatian. J Voice 2013;27(1):130. e7-130.e14

15 Hirano M. Clinical Examination of Voice. New York, NY: Springer; 1981:81-84

16 Kurtzke JF. Rating neurologic impairment in multiple sclerosis: an expanded disability status scale (EDSS). Neurology 1983;33(11): 1444-1452

17 Aronson A, Bless D. Clinical Voice Disorders. New York, NY: Thieme; 2009:73-97

18 Peyvandi A, Naghibzadeh B, Ahmady Roozbahany N. Neuro-otologic manifestations of multiple sclerosis. Arch Iran Med 2010; 13(3):188-192

19 Thomas FJ, Wiles CM. Dysphagia and nutritional status in multiple sclerosis. J Neurol 1999;246(8):677-682

20 Wessa P. Free Statistics Software. Office for Research Development and Education, version 1.1.23-r7. 2012. Available at: http://www. wessa.net/. Accessed 14.04.2012. 